\title{
Cognitive therapy training for psychiatrists
}

\section{Jan Scott \& Steven Moorhead}

Psychological therapies are a necessary component of any mental health service. Psychiatrists will require a working knowledge of different psychological approaches and many will wish to develop clinical skills to practice at least one model of psychotherapy. Historically, most psychiatry training schemes have offered exposure to psychodynamic psychotherapies, while training in other approaches has been less systematic. The expansion of research into brief psychological therapies and a wealth of recent publications on the subject have led to an increasing awareness of the benefits of training in other models of psychotherapy. The most widely practised and researched brief psychological intervention is cognitive-behavioural therapy (CBT). This paper will outline the principles behind providing training in $C B T$, and the barriers to successful implementation of a training programme.

\section{Training in cognitive- behavioural therapy}

CBT is a brief, structured, problem-oriented therapy with proven efficacy in the treatment of axis I disorders (Scott, 1995). Importantly, it can be used as an alternative to medication for individuals who cannot or do not wish to take psychotropic drugs. As such, CBT is a mainstream treatment which is potentially applicable to $60 \%$ of cases seen in psychiatric out-patient clinics (Stern, 1993). Its open, collaborative style, with shared decision-making and treatment-planning between patient and therapist give it additional appeal in an era of user empowerment. As CBT is a time-limited therapy, it also offers some prospect of increasing the availability of psychological interventions at a time when mental health services in general and psychotherapy services in particular are under increasing pressure to provide 'talking therapies' to a wider audience.

At an individual level, psychiatrists may want exposure to CBT for several reasons:

(a) Junior trainees may want teaching in CBT so that they can answer questions posed in the MRCPsych examinations or meet the requirements of the College guidelines (Royal College of Psychiatrists, 1993).

(b) Others will want to understand enough about cognitive models to allow them to make informed decisions about when or if to make a referral to an expert in CBT.

(c) Many psychiatrists will wish to develop their clinical competency so that they can apply CBT techniques in day-to-day practice.

(d) A minority may wish to develop a special interest in CBT or become cognitive psychotherapists.

Those involved in clinical practice or research in brief psychotherapies will want to encourage psychiatrists to develop clinical skills in CBT for four further reasons:

(a) There is an increasing trend toward the development of guideline-driven psychotherapies. Psychiatrists would benefit from clinical exposure to one such model at an early stage of their career to provide them with first-hand experience of the strengths, weaknesses and complexities of trying to implement a brief, operationalised programme of interventions.

(b) In an era of evidence-based medicine, psychiatrists would benefit from a practical as 
well as a theoretical understanding of the core characteristics shared by CBT and other effective psychological therapies (Scott, 1995: see Box 1).

(c) Many treatment studies claim to use CBT. Psychiatrists need to be able critically to appraise whether the psychological intervention described meets their own understanding of how CBT is practised, and whether the therapy provided was of sufficient quality to make the study results meaningful.

(d) In order to increase the CBT knowledge base of all psychiatrists and the clinical expertise of a more select group, there is a need to increase the number of psychiatrists with the required level of clinical competence to supervise novice therapists. In 1993, only two of 114 medically qualified psychotherapists identified themselves as cognitive therapists.

\section{Effective training models}

\section{Basic knowledge and understanding}

In order for a junior trainee to pass the MRCPsych examination or for a clinician to be able to make appropriate referrals for CBT, they will need (at a minimum) to develop a basic knowledge of the theory, research and practice of CBT. These sessions could comprise didactic lectures but interactive, student-centred, seminar-based teaching with specific learning objectives is preferable as this resonates more clearly with the philosophy underlying CBT (Drummond, 1992). Participants should be offered a comprehensive overview of different cognitive models, so that they understand that the theory and practice of CBT with different axis I disorders will vary and also that the treatment of axis I syndromes is different from the 'schemabased' CBT approach to personality disorders. This

Box 1. Shared characteristics of effective brief psychotherapies

Coherent theoretical model

Structured approach, with interventions based on case formulation

Emphasis on skills development and independent use of skills outside therapy

Change attributed to the person, not the therapist group teaching should be supplemented by relevant reading (Scott, 1994). The sessions should also include a detailed discussion of current debates about the assessment of suitability for CBT and the limitations of this form of therapy (Saffran \& Segal, 1990; Roth \& Fonagy, 1996).

On its own, the above teaching format rarely gives the trainee more than a crude understanding of the art of CBT. Indeed, because the theory underlying CBT is highly understandable, this approach has significant disadvantages, as it often perpetuates the myth that the therapy must likewise be easy to undertake. Unfortunately, knowledge expressed in writing does not predict clinical skilfulness (Perris, 1994). The 'minimum teaching module' should therefore include some exposure to practice. This approach dispels any fantasies that CBT has no concern for the therapeutic relationship and can be undertaken in a prescriptive manner by following a 'cook-book' of techniques. It also undermines reductionist arguments that CBT is a superficial therapy targeted only at symptoms rather than underlying processes and beliefs. Clinicians who believe that they are practising CBT because they help people to establish links between thoughts and feelings or to challenge negative automatic thoughts, rapidly realise that undertaking the therapy is a very different proposition from utilising cognitive or behavioural techniques in an ad hoc or opportunistic way.

To help a person successfully complete a course of CBT requires considerable skill and expertise on the part of the clinician. The therapist must be able to maintain a collaborative, empathic, therapeutic relationship while selecting specific interventions based on the cognitive conceptualisation of that individual's unique presentation. To achieve this, the therapist uses a process of guided discovery (not persuasion!) to engage the person in a dialogue about their beliefs and experiences, so that they can understand and 'own' the formulation. The individual can then play an active role in designing cognitive or behavioural experiments that address the specific problems and symptoms they have identified in a logical and coherent way. The therapist also needs to be able to judge when the person has developed the skills required to allow joint exploration and therapeutic work on more complex issues relating to underlying beliefs. If novice therapists are to develop any awareness of these aspects of CBT, they need opportunities to observe sessions of CBT undertaken at different stages in a course of therapy and to practice and experience some aspects of the process of CBT.

Observation of CBT may be 'live' by sitting in on sessions, or may be achieved by watching 
video-taped recordings of $C B T$ sessions. The advantage of the latter is that tapes may be made by local experts or may be purchased from recognised training centres and can form part of a readily accessible library. Trainees benefit greatly from small group discussions of therapy and the opportunity to pause and replay sections of videotapes. Access to written transcripts of the therapy videotapes also aids understanding. Practice and experience of CBT may be acquired through participation in workshops or seminars where experienced therapists can 'model' the therapy and clinicians may then engage in 'role plays' of clinical scenarios with colleagues. Experience suggests that opportunities to play the role of the patient are as important as playing the therapist. Many clinicians struggle to identify their automatic thoughts and the associated emotions and gain an understanding of how difficult it might be for patients to engage in CBT. Similarly, only by playing the therapist do they gain experience in trying to implement CBT strategies in a sensitive and systematic manner.

\section{Developing clinical skills}

The style of CBT is well suited to skills-based training and encourages clinicians to work towards explicit (and measurable) objectives. Although one of the most cost-efficient ways to offer training in CBT is through workshops, it is important to use this approach with caution. A series of regular workshops on a single cognitive model (depression) or on different styles of CBT (for example Beckian or constructivist approaches) can build up a trainee's knowledge and skills base to the level at which they could begin practising CBT (preferably with supervision). Single or irregular workshops for trainees or consultant CPD programmes, are of limited use if the goal is to develop the competent use of therapy skills by a novice therapist. Such a brief exposure to a different model of therapy may add to the repertoire of an experienced psychotherapist, but few other clinicians should leave an introductory workshop and practice CBT unsupervised. Single training sessions are best used to give a flavour of the therapy, to help psychiatrists determine who might be referred for CBT, or to teach clinicians one or two interventions that they could include in an eclectic treatment package with selected people.

More systematic approaches to CBT training require regular input and supervision from expert therapists and are rarely available in the UK or Republic of Ireland. There are essentially four models: regular supervision sessions, training clinics, post-qualification training courses (PQTCs), or specialist registrar training posts. The common element to all of these approaches is that the novice therapist has the opportunity to practice CBT and to gain first-hand experience through case supervision. High-quality CBT supervision essentially models the therapy relationship and process. The principle of 'collaborative empiricism' that is so central to CBT provides a solid basis on which to build a supervision relationship characterised by mutual trust, respect and understanding, while the use of Socratic questioning (guided discovery) promotes the trainee's active participation in the search for more appropriate therapeutic behaviours (Perris, 1994; see Box 2.)

Brittlebank \& Owens (1997) report on the benefits to a number of senior house officers in psychiatry

Box 2. Basic goals and stages of supervision (adapted from Perris, 1994)

\section{Basic goals}

To reach, as soon as possible, a conceptualisation of the problems of the person, which takes into account dysfunctional cognitions, emotions and behaviours, and points to the identification of underlying mechanisms

To establish a solid therapeutic relationship promoting an experience of security in the person and enhancing engagement

To define explicitly the goals to be attained and to adhere to the therapy programme

To select and appropriately apply in a timely manner those therapeutic strategies and techniques best suited to achieving the predefined goals

To decide what indicates to the therapist that a given goal has been attained, allowing a critical evaluation of the results of therapy

To recognise and evaluate the interpersonal reactions (in the individual and therapist) occurring during sessions, and understand how to deal with them

Stages of supervision

Stage 1: Focus on the person and practical conduct of therapy

Stage 2: Take into account (from CBT perspective) the interactions between the patient and therapist

Stage 3: Take into account (from CBT perspective) the interactions between the therapist and supervisor 
who were offered regular case supervision by a consultant with expertise in CBT. In many regions, this model may be the most simple to replicate, although it is dependent on an enthusiastic trainer being willing and able to offer CBT input. Training clinics usually draw on the skills (and goodwill) of three or four CBT experts and offer trainees the opportunity to take on 2-4 people for CBT and to participate in weekly peer group supervision, monthly presentations and an academic journal club. In Newcastle, the clinic is multi-disciplinary and held weekly for half a day (Scott et al, 1985). Trainees are expected to commit themselves to the clinic for one year. The weekly training session follows a set format, with trainees seeing a person selected for them from referrals to the CBT centre, attending small group supervision sessions $(n=4-$ $5)$ and then joining the whole group $(n=20)$ for presentations or academic sessions. Assuming a 'critical mass' of CBT experts exist, the three main practical difficulties associated with this approach are that there is a waiting list to join the clinic, that the clinic runs on a fixed day of the week so that trainees (usually at senior house officer level) have to negotiate time off from both their current and future training posts, and that (unlike most psychodynamic psychotherapy training schemes) permission to attend is not automatically granted.

A more sophisticated approach to CBT training is to provide a one-year PQTC. A number of teaching centres (Newcastle, Oxford, London and Manchester) have established diploma courses, with more beginning in Scotland, Northern Ireland and the Republic of Ireland. The courses are usually multi-disciplinary and accept about 20 individuals who have some previous experience of CBT. The training includes a mix of academic and clinical work with intensive weekly supervision. Trainees are often 'matched' with supervisors so that the clinical experience is tailored to the individual's expressed needs and training objectives. Trainees usually video- or audio-tape CBT sessions, which are then discussed in supervision and used to allow independent ratings of therapeutic competency. Weekly half-day workshops further develop general and specific CBT skills.

There are about five dedicated CBT specialist registrar training posts currently approved by the College. These offer extended experience in CBT, but also include at least 12 months' exposure to psychodynamic psychotherapy. This enables the trainee to put their CBT experience in context, to develop basic psychodynamic therapy skills and to clarify their ideas about which psychotherapy might be most appropriate for whom. The benefits of these posts will partly be judged on the opportunities for these specialist registrars to take up substantive consultant posts in CBT in the future, so increasing the psychotherapy options available to patients and the number of psychiatrists available to train junior doctors.

\section{Establishing competency}

Schaffer (1982) identified the relevant dimensions of therapist behaviour (see Box 3). Quality of any particular therapist's behaviour was more important than the quantity. Performance on each of these dimensions shows some correlation with patient outcome, but studies measuring multiple dimensions of therapist behaviour give a more accurate picture of therapist competency. Schaffer (1982) commented that trainees tend to neglect one or more dimensions unless supervised by a skilled expert in the therapy being used. He also noted that observation of therapist behaviour is the best way to assess competence.

Valliset al (1986) drew upon their work with CBT and depression to put together the Cognitive Therapy Rating Scale (CTRS). The CTRS comprises 13 items which relate to the three dimensions identified by Schaffer (1982) but which are divided between three sub-scales: interview process (e.g. agenda setting), general therapeutic skills (e.g. empathy, interpersonal effectiveness) and specific CBT skills (e.g. empiricism, focus on key cognitions, homework). Each item is rated on a 0-6 Likert scale

Box 3. Dimensions of therapist behaviour

Type-Refers to goals of behaviour and tactics for accomplishing these goals that are prescribed by the cognitive theory behind the therapy. For example, were homework assignments identified?

Skilfulness - refers to the extent to which the tactics are competently undertaken. Did the therapist collaborate with the person when setting the homework, was the introduction of the assignment timely and were the goals clearly identified and understood by patient and therapist?

Interpersonal manner-refers to the manner in which the therapist relates to the individual. Was the intervention undertaken within the context of a warm and empathic relationship? 
with higher scores indicating greater therapist competency. A score of less than $50 \%$ (39 out of a maximum 78) indicates inadequate delivery of CBT. This may arise because of 'serious protocol deviations' where the therapist fails to practice CBT (in the early stages of training novices often revert to practising models of psychotherapy they are familiar with), or low scores may indicate that although the therapist shows fidelity to the cognitive model the overall performance is poor (Shaw, 1984).Shaw (1984) demonstrated that a novice therapist often fails to meet 'minimum criteria' $(50 \%)$ on the CTRS for their first few people, but that they gradually develop their CBT skills over the next few until only about one in five sessions fail to meet the accepted standard. With regular supervision (minimum of fortnightly), ratings on the CTRS tend to plateau out after treating about four people, thus providing a reasonable indication of the therapists future level of expertise. Shaw also showed that CBT skills were significantly correlated with general psychotherapy skills $(r=0.7$; $n=606 ; P<0.001$ ).

It is hypothesised that clinicians who become competent CBT practitioners are comfortable with short-term models, are easily able to establish a problem-oriented alliance and are able to add 'technical' CBT skills to their general psychotherapy skills (Shaw, 1984; Roth \& Fonagy, 1996; Scott, 1996). Novice CBT therapists initially favour one or two techniques over all others, and only gradually increase their repertoire. Only later do they acquire strategic skills. Conceptualisation skills and more formal practice of CBT as a therapy rather than as a collection of techniques comes later still. This description fits with other models described by educational researchers. Five levels of skills acquisition are recognised: novice, advanced beginner, competent, proficient and expert. This approach is helpful when assessing a therapists' level of skill (see Table 1). The training packages described above all aim to develop clinicians to level three. However, many CBT trainers report that long-term supervision is required to maintain CBT therapists at this level or to move them on to proficient or expert levels of practice. In Newcastle, we have noted that without maintenance supervision, many therapists show 'recidivism', reverting to their previously favoured therapy approaches or diluting their CBT style with a 'mixed therapy' approach. If regular supervision cannot be provided with a senior CBT therapist, trainees are encouraged to link up with an identified 'buddy' for peer supervision, or to listen regularly to audiotapes of their therapy sessions to monitor their adherence to a CBT model and to retain a rigorous approach. We also offer a monthly CBT 'forum' where experienced therapists meet and discuss case material or other clinical issues.

\section{Training makes a difference}

A number of research studies have assessed the relationship between therapists' competency in CBT and the individual's outcome. DeRubeis \& Feeley (1990) demonstrated a significant correlation $(r=0.5)$ between the therapist's adherence to the CBT model and outcome. In a study of 185 individuals treated by senior CBT therapists ( $>4$ years CBT experience) it was found that they were less likely to drop out of therapy and showed significantly greater improvement at the end of treatment than those treated by novice therapists (Burns \& Nolen-Hoeksema, 1992). Further investigation has established that it is expertise rather than simply length of exposure to CBT that is critical, with therapists' level of skill contributing 20-30\% of the variance in patient outcome (Roth \& Fonagy, 1996; Scott, 1996). Therapeutic expertise is particularly important when treating more severely disturbed people (Roth \& Fonagy, 1996; Scott, 1996). These findings highlight the importance of assessing the therapist's competence when reviewing a person's response or non-response to CBT in research or clinical settings.

\section{Overcoming training barriers}

Professional rivalries, resource issues and practical problems may all impede opportunities for training in CBT. It would be foolish to pretend that there is universal approval for the principle of training psychiatrists in CBT. Many psychologists and some nurses feel that psychiatrists should focus on their traditional roles. Stern (1993) argues that if psychiatrists do not train in CBT they will be left therapeutically impotent and less able to lead a multi-disciplinary team. However, the lack of trained psychiatrists means that we often need to approach non-medical experts in CBT to offer training. Given the limited resources available, many of these professionals argue that they should prioritise the needs of trainees from their own discipline before offering input to psychiatrists. The alternative approach is to increase the number of psychiatrists trained in CBT by increasing the availability on training schemes and increasing the number of specialist registrar and consultant posts. However, in a time of scarce resources, there are tensions within the psychotherapy world about how this is best achieved. It must be borne in mind that CBT is a relatively new therapy with no 
established 'home' within the College. While CBT clearly shares many agenda with psychotherapy, the application of CBT to mainstream psychiatric practice raises intriguing questions about whether its long-term future might be within general adult psychiatry.

At a practical level, there are three obvious issues that need to be addressed:

(a) Even if resources are available to train psychiatrists in CBT, should trainees be exposed to psychodynamic and cognitive approaches simultaneously or sequentially (and if so in what order)? Simultaneous training in both psychodynamic and CBT approaches would probably be unrealistic in most training schemes and counterproductive for most trainees, as few novice therapists could develop competence in both approaches at the same time. The need to have established general psychotherapy and interpersonal skills and the well-established programmes of training in psychodynamic therapy argue that these approaches might best be given precedence. However, anecdotal evidence from training courses in the USA and England suggest that it is more difficult to change from psychodynamic to cognitive models of practice than from behavioural to cognitive models of practice. Henryet al (1993) offer tentative support for this view, as they reported that when psychodynamic therapists are asked to practice guidelinedriven therapies some develop technical adherence at the expense of their interpersonal transactions. How to provide training in both approaches or whether an either/or approach is required clearly needs further discussion.

(b) It may be helpful to review the College guidelines on training in CBT, which suggest that trainees should take on two short cases and one long case. The CBT approaches required in these situations are rather different and even fewer trainers are available to supervise 'schema-based' CBT. Furthermore, the treatment of four short cases seems to be the most effective way to establish competency in CBT (Shaw, 1984).

(c) Training opportunities for senior psychiatrists should take into account that while the CBT trainers may be experts in this approach they will usually be younger, and less experienced

Table 1. Ievels of therapist competency

Skills level Clinical behaviour

Level 1:

Novice

Level 2:

Advanced

beginner

Level 3:

Competent

practitioner

Level 4:

Proficient

practitioner

Level 5:

Expert practitioner
Rigid adherence to taught techniques

Little overall strategy

Poor judgement in choice of target problem

More judgement in choice, but still favours 1-2 techniques

Overall strategy still limited

All presenting problems given equal importance

Choice of technique appropriate to identified problem

More systematic planning of interventions

Structure of therapy follows a standard and routine pattern

Sees need for interventions to be part of longer-term strategy and case formulation (cannot always achieve this)

Is guided by the case formulation in choice of target problems and type of interventions

Discerns what is most important in communication and more aware of unique needs or characteristics of the person

Therapy proceeds smoothly, decision-making is less laboured

Develops sophisticated and accurate case conceptualisation

Focuses on important aspects of problems

Uses standard techniques smoothly and is able to repair

ruptures in the therapeutic relationship

Has intuitive grasp and deep tacit understanding of the individuals problems

No longer adheres rigidly to guidelines, able to use technical recommendations flexibly and deviates or goes beyond them when the clinical situation requires this
Training process

Seminar-based teaching Irregular exposure to workshops

More regular workshops

Basic supervision

Training clinic

Training clinic (a minority will be able to reach this standard in this setting)

Regular supervision PQTC

PQTC with post-course maintenance supervision Specialist registrar training post in CBT

Continuous experience and practice of CBT ( $>4$ years) with regular peer supervision and frequent attendance at CPD and training workshops 
in psychiatry, than their trainees (Stern, 1993). The situation must be handled with sensitivity to ensure that the experience is a positive one for both parties.

\section{References}

Burns, D. \& Nolen-Hoeksema, S. (1992) Therapeutic empathy and recovery from depression in cognitive therapy: A structural equation model. Journal of Consulting and Clinical Psychology, $60,441-449$.

Brittlebank, A. \& Owens, J. (1997) CBT training for junior psychiatric trainees. Psychiatric Bulletin, 21, 169-170.

DeRubeis, R. \& Feeley, M. (1990) Determinants of change in cognitive therapy for depression. Cognitive Therapy and Research, $14,469-482$.

Drummond, L. (1992) Behavioural and cognitive psychotherapy experience and supervision: A possible model of delivery. Psychiatric Bulletin, 16, 154-157.

Henry, W., Schacht, T., Strupp, H., et al (1993) Effects of training in time-limited psychotherapy: Mediators of therapists' response to training. Journal of Consulting and Clinical Psychology, 61, 441-447.

Perris, C. (1994) Supervising cognitive psychotherapy and training supervisors. Journal of Cognitive Psychotherapy, 8, 83-103.

Roth, A. \& Fonagy, P. (1996) What Works for Whom? A Critical Review of Psychotherapy Research. London: Guilford Press.

Royal College of Psychiatrists (1993) Guidelines for Psychotherapy Training as Part of General Professional Training. Council Report CR27. London: Royal College of Psychiatrists.

Saffran, J. \& Segal, Z. (1990) Interpersonal Process in Cognitive Therapy. New York: Basic Books.

Schaffer, N. (1982) Multi-dimensional measures of therapist behaviour as predictors of outcome. Psychological Bulletin, 92, 670-681.

Scott, J. (1994) Reading about cognitive therapy. British Journal of Psychiatry, 165, 410-415.

- (1995) Psychological treatment of depression: An update. British Journal of Psychiatry, 167, 289-292.

- (1996) Cognitive therapy of affective disorders: A review. Joumal of Affective Disorders, 37, 1-11.

-, Barker, W. \& Williams, J. M. G. (1985) The teaching of cognitive therapy in Newcastle. Psychiatric Bulletin, 9, 33-34.

Shaw, B. (1984) Specification of the training and evaluation of cognitive therapists for outcome studies. In Psychotherapy Research: Where Are We and Where Should We Go? (eds J. Williams \& R. Spitzer), pp. 92-128. London: Guilford Press.

Stern, R. (1993) Behavioural-cognitive psychotherapy training for psychiatrists. Psychiatric Bulletin, 17, 1-4.

Vallis, T., Shaw, B. \& Dobson, K. (1986) The cognitive therapy scale: psychometric properties. Journal of Consulting and Clinical Psychology, 54, 381-385.

\section{Multiple choice questions}

1. To become competent as a therapist in CBT, a trainee:

a only requires attendance at a series of seminars on different cognitive models

b should attend a series of seminars plus one or two clinical workshops

c should receive supervision on at least four people taken on for CBT

d should receive supervision at least once per fortnight.
2. When training as a cognitive therapist :

a trainees develop clinical strategy before they develop technical CBT skills

b trainees find it hard to attend simultaneously to the type, skilfulness and interpersonal manner of their interventions

c trainees should regularly rate their own performance as a therapist using the Cognitive Therapy Rating Scale

d it takes about four years to become an expert practitioner.

3. Process and outcome studies of CBT demonstrate:

a that there is no relationship between general psychotherapy and CBT skills

b that less than $20 \%$ of the variance in a persons' outcome is attributed to therapist skill

c that experience in CBT is a particularly important mediator in the outcome of more severe or chronic disorders

d that expert therapists achieve better outcomes because they apply CBT in a prescriptive manner. 